\title{
Lyotropic phase behaviour of dilute, aqueous hen lysozyme amyloid fibril dispersions
}

\author{
Christian Müller • Olle Inganäs
}

Received: 6 December 2010/Accepted: 31 January 2011/Published online: 11 February 2011

(C) Springer Science+Business Media, LLC 2011

\begin{abstract}
We explore the lyotropic phase behaviour of dilute, aqueous amyloid fibril dispersions from hen egg white lysozyme with respect to protein and acid concentration in order to establish preparation protocols that provide homogeneous nematic phases. Such ordered dispersions are demonstrated to facilitate alignment of amyloid nanofibrils in thin solid films, which are utilised to structure conjugated (poly)electrolytes. In addition, the occurrence of ordered phases is found to be in good qualitative agreement with phase equilibria predicted for dispersions of rod-like particles.
\end{abstract}

\section{Introduction}

Amyloid fibrils-highly anisotropic, $\beta$-sheet rich entities that arise from misfolded proteins - have attracted much attention as they have been associated with a variety of human diseases [1]. However, occasionally such structures have been found to perform benign biological functions in living organisms, for example as a matrix material that facilitates surface-binding of Escherichia coli bacteria [2], or as a catalytic scaffold for the synthesis of melanin in human melanosomes [3]. Inspired by such potentially useful qualities, more recently, amyloid fibrils have been extensively investigated as functional materials that direct

C. Müller $(\varangle) \cdot$ O. Inganäs

Biomolecular and Organic Electronics, Department of Physics, Chemistry and Biology (IFM), Linköpings Universitet,

58183 Linköping, Sweden

e-mail: christian.muller@icmab.es

Present Address:

C. Müller

Institut de Ciència de Materials de Barcelona (ICMAB-CSIC),

Esfera UAB, 08193 Bellaterra, Spain supra-molecular self-assembly [4-6], or serve as templates for, e.g. metal nanoparticles [7-9] and conjugated (poly)electrolytes [10-14]. Such prepared nanofibrils are of particular interest for applications in biotechnology and organic opto-electronics because of their characteristic feature sizes, i.e. up to several micrometres in length but only a few nanometres in diameter.

Previous efforts have been largely limited to the study of isolated nanofibrils or unstructured networks, whereas systems that comprise ordered collectives have been explored to a lesser extent. Conveniently, colloidal dispersions of high aspect ratio amyloid fibrils from polypeptides [15-18] and proteins such as insulin [19, 20], lysozyme [21, 22] or $\beta$-lactoglobulin $[23,24]$ display lyotropic ordering in the form of phase-separated spherulitic droplets or nematic liquids. Clearly, the liquid-crystalline nature of such systems may lend itself as a powerful tool to order these materials, e.g. through uniaxial alignment in thin films.

Thus, here we explore the phase behaviour of dilute, aqueous amyloid fibril dispersions from hen egg white lysozyme with respect to protein and acid concentration in order to establish preparation protocols that yield suitable lyotropic phases. Accordingly, we demonstrate that the use of homogeneous nematic liquids readily permits alignment of the $\beta$-sheet material in thin solid films, which for instance may serve as templates for opto-electronically active conjugated (poly)electrolytes.

\section{Experimental}

\section{Materials}

Hen egg white lysozyme was purchased from SigmaAldrich and used as received (product number L6876; 
additional supplier information: dialysed, lyophilised, three times crystallised, contains sodium acetate and sodium chloride buffer salts, protein content $\geq 90 \%$ ). Tap water was distilled with a MilliQ purifier and aqueous $1 \mathrm{M}$ hydrochloric acid ( $\mathrm{HCl}$; Sigma-Aldrich) was added to reach the desired acid concentration. Congo Red was obtained from Serva Feinbiochemica, Heidelberg and poly(thiophene acetic acid) (PTAA) had been prepared according to a previously recorded procedure [25].

\section{Sample preparation}

Colloidal dispersions of amyloid fibrils were produced by incubating solutions of the native protein in aqueous $\mathrm{HCl}$ for 2 weeks at $65{ }^{\circ} \mathrm{C}$ in a closed hot oven to guarantee a homogeneous temperature environment. Solutions were kept in closed polypropylene vials (2 $\mathrm{mL}$ volume) that were tightly sealed with PTFE tape to prevent evaporation. [Note that, for unknown reasons, amyloid formation appeared to proceed at a slower rate if solutions were incubated in glass vials.]

Aligned thin films were prepared by doctor-blading concentrated amyloid dispersions on glass microscopy slides, which was achieved by shearing the dispersion with a razor blade that was held in close proximity to the glass surface. Amyloid films were stained with $1 \mathrm{wt} \%$ Congo Red (or PTAA) aqueous solution after drying; excess dye was removed by rinsing with distilled water.

\section{$\mathrm{pH}$ determination}

The $\mathrm{pH}$ of dispersions was determined with a Biotrode $\mathrm{pH}$ meter (Mettler Toledo).

Transmission electron microscopy (TEM)

TEM was performed with a Philips CM200 microscope, operated at $200 \mathrm{kV}$. Samples were prepared by depositing diluted amyloid dispersions $(\sim 0.5 \mathrm{mM}$ with respect to initial protein concentration) on 200 mesh carbon-coated copper grids (Agar Scientific) and, after drying, stained with $2 \mathrm{wt} \%$ aqueous uranyl acetate solutions (three times for $10 \mathrm{~s}$ followed by rinsing with distilled water; SigmaAldrich).

\section{Polarised optical microscopy}

Optical microscopy was carried out with an Olympus BH2 polarising microscope. Wet samples were sandwiched between glass microscopy and cover slides.
Ultraviolet-Visible light (UV-Vis) absorbance spectroscopy

Polarised UV-Vis absorbance spectra were recorded using a Perkin Elmer Lambda 950 UV-Vis spectrophotometer.

\section{Results and discussion}

Preparation of amyloid nanofibrils

Amyloid fibril dispersions from hen egg white lysozyme in aqueous hydrochloric acid $(\mathrm{HCl})$ were prepared at previously established growth conditions, i.e. at a $\mathrm{pH}$ between 1 and 3 and a temperature of $65{ }^{\circ} \mathrm{C}[21,22,26,27]$. These conditions are ideal for amyloid formation because this range of $\mathrm{pH}$ is situated far below the isoelectric point of lysozyme $(\mathrm{p} I \sim 11) .{ }^{1}$ The high positive net charge of the protein results in long-range repulsion, which permits the slow growth of regular $\beta$-sheet structures. Instead, incubation close to the $\mathrm{p} I$ would lead to rapid aggregation and thus the formation of irregular particulates [28].

Irrespective of the acid and protein concentration, nanofibrillar structures with a diameter of approximately $5 \mathrm{~nm}$ and a length in excess of one micrometre could be observed after subjecting protein solutions to elevated temperature, as evidenced by a series of transmission electron micrographs in Fig. 1. Nevertheless, the nucleation and growth rate of amyloid fibrils is likely to vary with composition and $\mathrm{pH}[26,27]$, which may affect in particular their length distribution but also internal structure. For instance, based on a theoretical study, it has been argued that the length distribution of hen lysozyme amyloid fibrils depends on the initial protein concentration [29]. Hydrolysis of lysozyme can also occur, especially at $\mathrm{pH}<3$ and elevated temperature, but both the full length protein as well as its fragments are incorporated into amyloid fibrils [27]. In addition, it should be noted that despite the presence of numerous amyloid fibrils, it cannot be ruled out that a fraction of the initial protein remains present even after 2 weeks of incubation.

\section{Lyotropic phase behaviour}

In order to elucidate the appearance of anisotropic phases, we examined amyloid dispersions with polarised optical microscopy (Fig. 2a). At dilute protein concentration, e.g. less than $0.2-0.7 \mathrm{mM}$ initial lysozyme content, no birefringence was observed, indicating homogeneous isotropic dispersions, in agreement with a previous study [21].

\footnotetext{
${ }^{1}$ www.expasy.ch/tools.
} 
Fig. 1 Transmission electron micrographs of dried, uranyl acetate-stained amyloid fibrils grown at various lysozyme and $\mathrm{HCl}$ concentrations. The acid concentration is expressed as $-\log [\mathrm{HCl}]$ in order to more closely relate to the actual $\mathrm{pH}$ of investigated samples

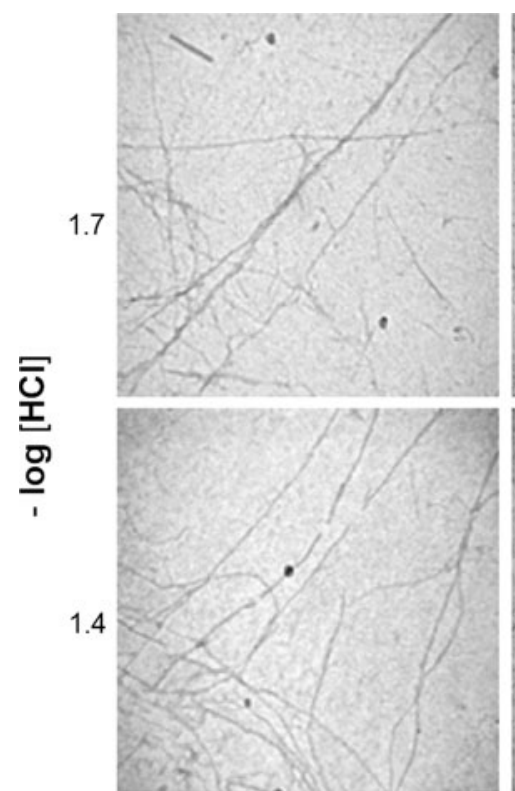

0.5

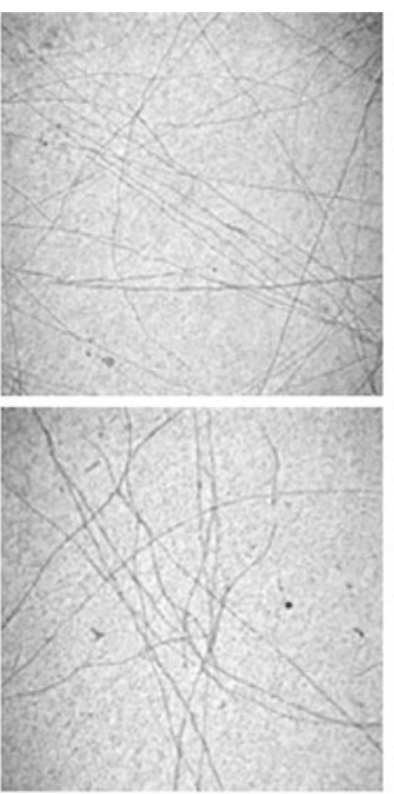

1

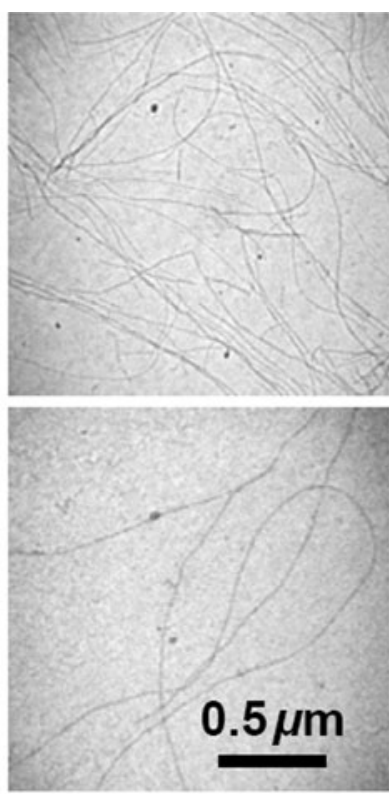

2

lysozyme (mM)

In stark contrast, at intermediate concentrations around 0.7-1.4 mM, birefringent spherulitic structures suspended in an isotropic liquid had developed, which previously have been associated with $\beta$-sheet fibrils radially grown from a more disordered nucleus [20]. In addition, for sufficiently acidic dispersions, i.e. for acid concentrations of $\sim 30 \mathrm{mM}$ $\mathrm{HCl}$ and above (corresponding to $-\log [\mathrm{HCl}] \leq 1.5$; c.f. Fig. 2), a heterogeneous distribution of fibrillar material in the form of spherulitic domains was found to extend to at least $4 \mathrm{mM}$ initial lysozyme concentration. Here, it is critical to note that with increasing protein content spherulites ceased to be surrounded by an isotropic medium but, instead, were dispersed in a more birefringent liquid, which due to limitations of our imaging equipment is not apparent from the optical micrographs displayed in Fig. 2a. Less acidic dispersions, in contrast, featured a homogeneous nematic texture above a lysozyme concentration of $1.5 \mathrm{mM}$. However, spherulitic domains were not found to develop, when diluting nematic liquids, which instead separated into isotropic and anisotropic fractions, confirming that the spherulitic nature is particular to phaseseparation during amyloid formation [20]. This is also consistent with our observation that spherulites did not form in protein solutions that were subjected to vortexing during incubation, which appeared to disrupt the formation of regular structures and gave rise to disordered birefringent aggregates instead, as also reported for amyloid fibrils from bovine insulin [30].

Interestingly, the distribution of homogeneous isotropic, homogeneous anisotropic and heterogeneous regions (i.e. the coexistence of either an isotropic and lyotropic phase or two lyotropic phases) is in good qualitative agreement with phase equilibria predicted for solutions of rod-like particles (Fig. 2b) [31], which is astonishing considering the fact that samples were prepared at vastly different incubation conditions. The narrow isotropic, biphasic and extended nematic region at low acid concentration, i.e. $-\log [\mathrm{HCl}]>1.5$, result from appropriate volume fractions of rigid rod-like amyloid fibrils, as predicted by Onsager theory [32], which is thoroughly discussed in Ref. [23]. In contrast, the transition from a homogeneous to a heterogeneous distribution of material around $-\log [\mathrm{HCl}]=1.5$ can be understood in terms of the change in electrostatic interaction of amyloid fibrils with the aqueous dispersion medium, which may-at least in part-give rise to the observed phase behaviour. Previous studies of amyloid fibrils from $\beta$-lactoglobulin or bovine insulin have revealed that the propensity for aggregation is related to the electrostatic repulsion between fibrils, which could be altered through partial screening. This was accomplished by varying the ionic strength of the dispersion medium [23, 30], as well as by adjusting the $\mathrm{pH}$, resulting in aggregation around the isoelectric point, at which fibrils carry no net electric charge [24]. Similarly, for the here investigated system it appears that at high acid concentration and thus presence of a high amount of chloride ions, similar screening effects or, potentially, salt bridges led to formation of heterogeneous structures; interactions, which in another report were found to become significant for peptide-based $\beta$-sheet fibrils at $\mathrm{pH} \leq 2$ [16]. Hence, the tendency to develop heterogeneous textures only dominates at sufficiently low $\mathrm{pH}$, i.e. at $\mathrm{pH}<1.7-1.9$, 


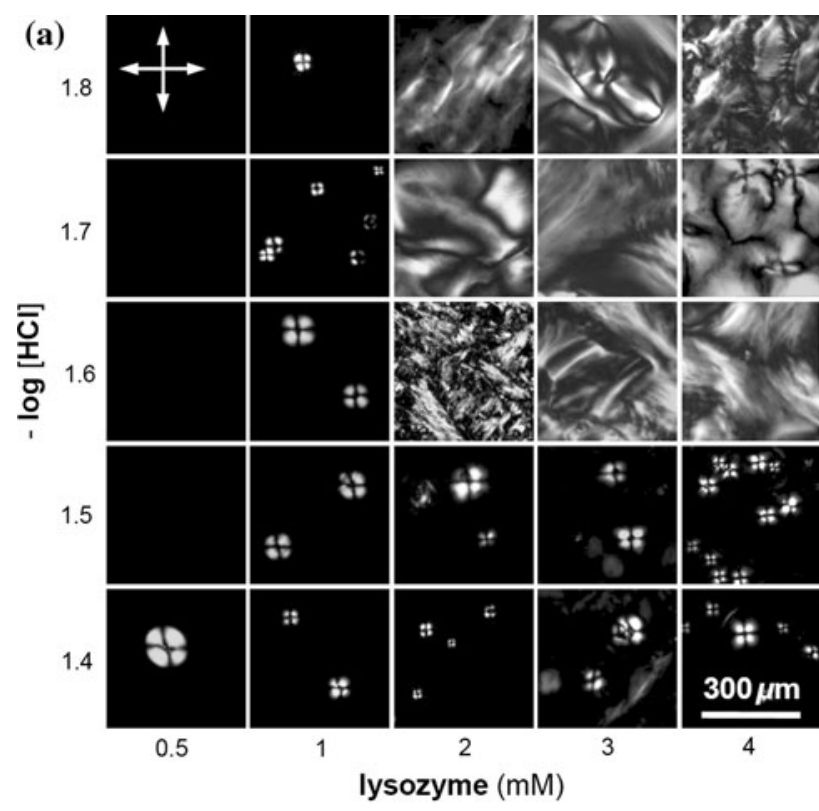

(b)

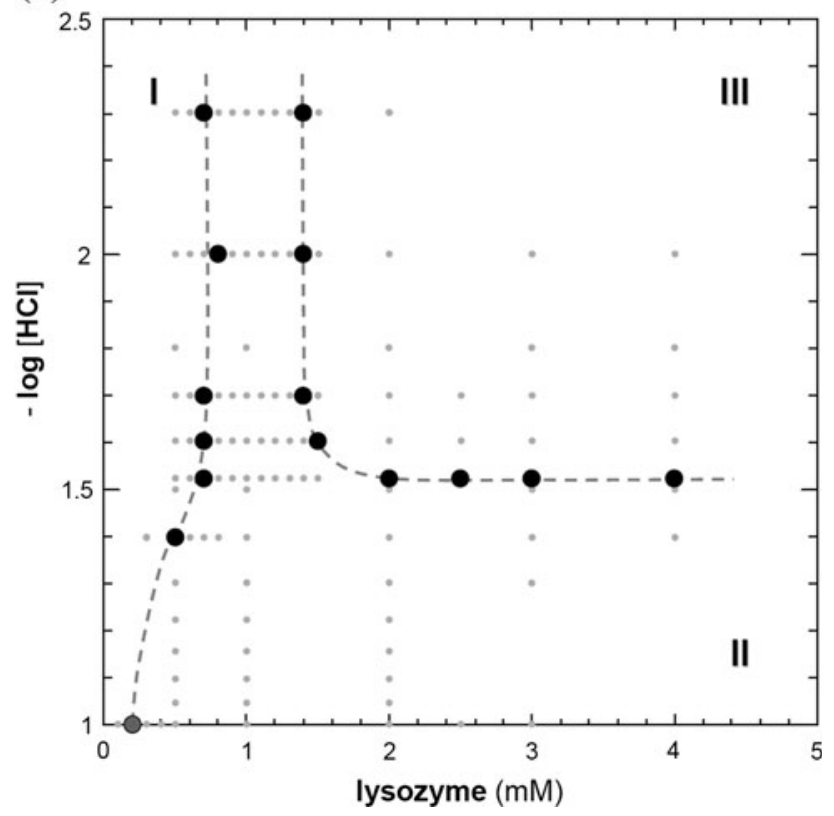

Fig. 2 a Polarised optical micrographs of amyloid fibril dispersions prepared at various lysozyme and $\mathrm{HCl}$ concentrations (orientation of polarisers indicated by crossed arrows). Note that we were unable to record all micrographs at the same light intensity because of the exceptionally strong birefringence of spherulitic structures. Therefore, for dispersions corresponding to $-\log [\mathrm{HCl}] \leq 1.5$ and an initial lysozyme concentration above $2 \mathrm{mM}$, it is not apparent that the liquid surrounding spherulites is, in fact, birefringent. b Occurrence of homogeneous isotropic $(I)$ and homogeneous anisotropic $(I I I)$ as well as heterogeneous textures $(I I)$ as revealed by polarised optical microscopy. Small circles represent investigated samples; large circles correspond to compositions, at which a transition from homogeneous to heterogeneous liquid-i.e. a liquid that contains spherulites-was observed

depending on the protein concentration (Fig. 3). It should also be noted that in the here investigated $\mathrm{pH}$ range, i.e. $\mathrm{pH}$ 1-3, aspartic and glutamic acid residues are likely to be

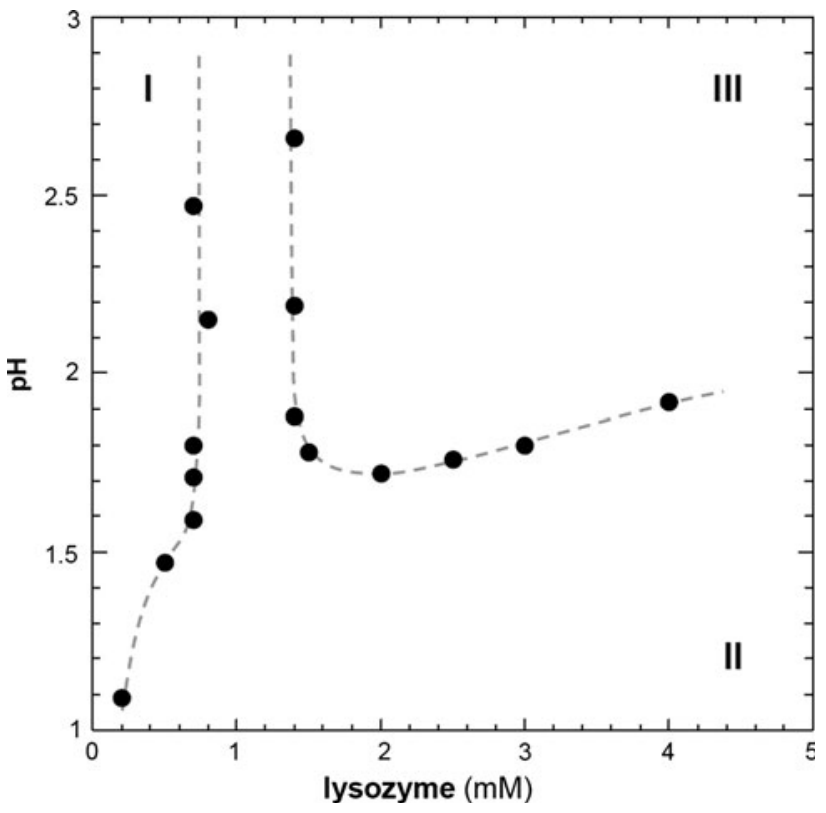

Fig. 3 pH/protein concentration phase diagram constructed with transitions from homogeneous isotropic $(I)$ or homogeneous anisotropic (III) to heterogeneous textures (II) that were deduced by polarised optical microscopy (c.f. Fig. 2b); the $\mathrm{pH}$ was estimated before amyloid formation

protonated and, therefore, the net electrical charge of lysozyme will be largely invariant.

Thin solid films order conjugated (poly)electrolytes

Heterogeneous dispersions, i.e. those that contain spherulites, don't easily permit uniaxial alignment of the nanofibrillar $\beta$-sheet rich material. In contrast, homogeneous isotropic dispersions have been shown to order when subjected to flow fields $[21,33]$, which can be retained through suitable surface interactions $[11,12]$. Homogeneous nematic dispersions instead, which readily feature local alignment of amyloid fibrils, usually displayed a high density of disclinations (c.f. Fig. 2a). However, largely defect-free thin solid films could be prepared through doctor-blading such liquids. Ordering was most visible when staining dried amyloid films with the conjugated electrolyte Congo Red, which resulted in pronounced apple-green birefringence when observed between crossed polarisers and gave rise to anisotropic absorbance as evidenced by polarised UV-Vis absorbance spectroscopy (Fig. 4). The absorbance maximum of the dye red-shifted from 502 to $507 \mathrm{~nm}$, confirming binding to $\beta$-sheets [26]. Previous studies have shown that Congo Red and therefore its dipole preferentially orient with respect to the long axis of amyloid fibrils [34-36], suggesting that here these structures tend to align with the shear direction during film deposition. 
(a)

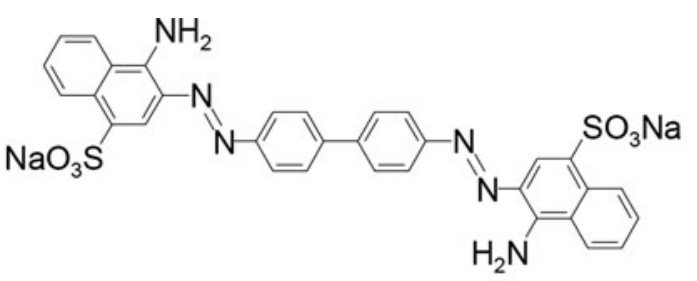

(b)
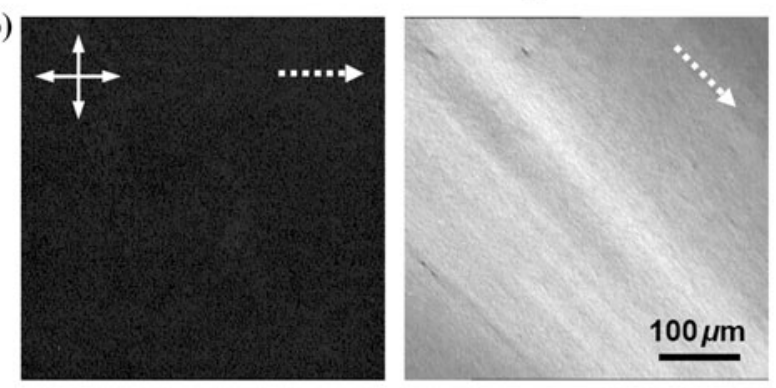

(c)

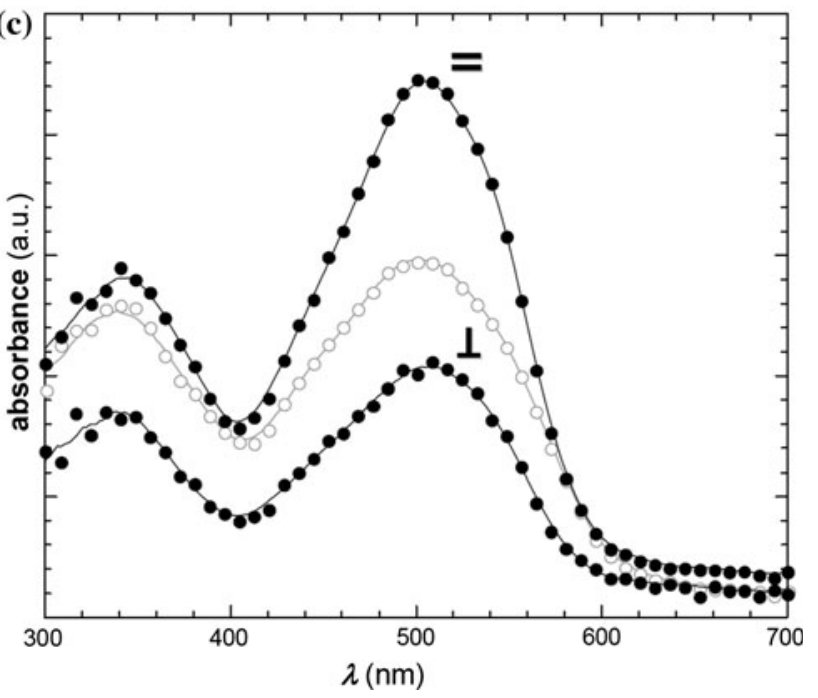

Fig. 4 a Chemical structure of Congo Red. b Polarised optical micrographs (orientation of polariser and analyser indicated by crossed arrows) of a typical Congo Red-stained thin solid film produced by doctor-blading and subsequent drying of a homogeneous nematic liquid. Amyloid fibrils were prepared at $2 \mathrm{mM}$ initial lysozyme content and $20 \mathrm{mM} \mathrm{HCl}$; shear direction parallel (left) and at $45^{\circ}$ with respect to the polariser (right) as indicated by dashed arrows. c Corresponding polarised UV-Vis absorbance spectra of the same Congo Red-stained film (black circles shear direction with respect to the polariser as indicated), as well as of reference Congo Red (white circles)

Likewise, staining with the conjugated polyelectrolyte poly(thiophene acetic acid) (PTAA) gave rise to maximum light absorbance when illuminated with light polarised parallel to the shear direction and displayed a shift in peak absorbance from 430 to $450 \mathrm{~nm}$ (not shown), in accordance with another report [11]. In contrast to studies on single polyelectrolyte-decorated amyloid fibrils [11, 12], we could only achieve marginal orientation of the conjugated moiety as evidenced by the relatively low dichroic ratios of our oriented films. Besides insufficient ordering of the template, this may have resulted from the commonly observed helical nature of lysozyme amyloid fibrils [21, 26, 27], which will give rise to misalignment of the bound dye and, hence, its absorbing dipole with respect to the shear direction. In principle, however, straight fibrils, e.g. grown from shorter peptides [17], may facilitate efficient ordering of conjugated electrolytes in thin films, provided homogeneous lyotropic dispersions can be prepared as outlined in this study.

\section{Conclusions}

In summary, we have shown that judiciously chosen nematic amyloid dispersions readily permit alignment of the nanofibrillar $\beta$-sheet rich material in thin solid films, which can be utilised to structure conjugated (poly)electrolytes. Furthermore, the occurrence of ordered phases was found to be in good qualitative agreement with phase equilibria predicted for dispersions of rod-like particles; an observation which may ease selection of suitable preparation protocols for similar lyotropic amyloid systems.

Acknowledgements We would like to acknowledge funding from the Swedish Foundation for Strategic Research (SSF) through the programme Organic hybrid Printed Electronics and Nanoelectronics (OPEN). Furthermore, we thank Prof. Mats Andersson (Chalmers tekniska högskola) for generously providing the PTAA used in this study.

\section{References}

1. Chiti F, Dobson CM (2006) Ann Rev Biochem 75:333

2. Chapman MR, Robinson LS, Pinker JS, Roth R, Heuser J, Hammar M, Normark S, Hultgren SJ (2002) Science 295:851

3. Fowler DM, Koulov AV, Alory-Jost C, Marks MS, Balch WE, Kelly JW (2006) PLOS Biol 4:100

4. Jahnke E, Lieberwirth I, Severin N, Rabe JP, Frauenrath H (2006) Angew Chem Int Ed 45:5383

5. Krysmann MJ, Castelletto V, McKendrick JE, Clifton LA, Hamley IW, Harris PJF, King SM (2008) Langmuir 24:8158

6. Channon KJ, Devlin GL, Magennis SW, Finlayson CE, Tickler AK, Silva C, MacPhee CE (2008) J Am Chem Soc 130:5487

7. Reches M, Gazit E (2003) Science 300:625

8. Scheibel T, Parthasarathy R, Sawicki G, Lin X-M, Jaeger H, Lindquist SL (2003) PNAS 100:4527

9. Malisauskas M, Meskys R, Morozova-Roche LA (2008) Biotechnol Prog 24:1166

10. Herland A, Björk P, Nilsson KPR, Olsson JDM, Åsberg P, Konradsson P, Hammarström P, Inganäs O (2005) Adv Mater 17:1466

11. Herland A, Björk P, Hania PR, Scheblykin IG, Inganäs O (2007) Small 3:318

12. Herland A, Thomsson D, Mirzov O, Scheblykin IG, Inganäs O (2008) J Mater Chem 18:126

13. Hamedi M, Herland A, Karlsson RH, Inganäs O (2008) Nano Lett $8: 1736$ 
14. Knowles TPJ, Oppenheim TW, Buell AK, Chirgadze DY, Welland ME (2010) Nat Nanotechnol 5:204

15. Aggeli A, Nyrkova IA, Bell M, Harding R, Carrick L, McLeish TCB, Semenov AN, Boden N (2001) PNAS 98:11857

16. Aggeli A, Bell M, Carrick LM, Fishwick CWG, Harding R, Mawer PJ, Radford SE, Strong AE, Boden N (2003) J Am Chem Soc 125:9619

17. Han TH, Kim J, Park JS, Park CB, Ihee H, Kim SO (2007) Adv Mater 19:3924

18. Hamley IW, Castelletto V, Moulton C, Myatt D, Siligardi G, Oliveira CLP, Pedersen JS, Abutbul I, Danino D (2010) Macromol Biosci 10:40

19. Waugh DF (1946) J Am Chem Soc 68:247

20. Krebs MRH, Bromley EHC, Rogers SS, Donald AM (2005) Biophys J 88:2013

21. Corrigan AM, Müller C, Krebs MRH (2006) J Am Chem Soc 128:14740

22. Yagi N, Ohta N, Matsuo T (2009) Int J Biol Macromol 45:86

23. Sagis LMC, Veerman C, van der Linden E (2004) Langmuir 20:924
24. Jung J-M, Mezzenga R (2010) Langmuir 26:504

25. Ding L, Jonforsen M, Roman LS, Andersson MR, Inganäs O (2000) Synth Met 110:133

26. Krebs MRH, Wilkins DK, Chung EW, Pitkeathly MC, Chamberlain AK, Zurdo J, Robinson CV, Dobson CM (2000) J Mol Biol 300:541

27. Arnaudov LN, de Vries R (2005) Biophys J 88:515

28. Krebs MRH, Devlin GL, Donald AM (2007) Biophys J 92:1336

29. Lee CF (2009) Phys Rev E 80:031902

30. Loksztejn A, Dzwolak W (2008) J Mol Biol 379:9

31. Flory PJ (1956) Proc R Soc London A 234:73

32. Onsager L (1949) Ann N Y Acad Sci 51:627

33. Adachi R, Yamaguchi K, Yagi H, Sakurai K, Naiki H, Goto Y (2007) J Biol Chem 282:8978

34. Romhányi G (1971) Virchows Arch Abt A 354:209

35. Klunk WE, Pettegrew JW, Abraham DJ (1989) J Histochem Cytochem 37:1273

36. Jin L-W, Claborn KA, Kurimoto M, Geday MA, Maezawa I, Sohraby F, Estrada M, Kaminsky W, Kahr B (2003) PNAS 100:15294 